\title{
A uniqueness result for final boundary value problem of microstretch bodies
}

\author{
M. Marin ${ }^{\mathrm{a}, *}$, D. Baleanu ${ }^{\mathrm{b}, \mathrm{c}}$, C. Carstea ${ }^{\mathrm{a}}$, R. Ellahi ${ }^{\mathrm{d}, \mathrm{e}}$ \\ ${ }^{a}$ Department of Mathematics and Computer Science, Transilvania University of Brasov, 500093 Brasov, Romania. \\ ${ }^{b}$ Department of Mathematics, Cankaya University, Ankara, Turkey. \\ ${ }^{c}$ Institute of Space Sciences, Magurele, Bucharest, Romania. \\ ${ }^{d}$ Department of Mathematics and Statistics, FBAS, IIUI, Islamabad 44000, Pakistan. \\ ${ }^{e}$ Department of Mechanical Engineering, University of California Riverside, USA.
}

Communicated by A. Petrusel

\begin{abstract}
Main subject of this study is the final boundary value problem of a microstretch thermoelastic body. In fact, using an elementary transformation, this problem is reformulated as a known mixed problem with initial and boundary conditions. We prove some results of uniqueness of solutions avoiding any conservation law of energy. We also give up any hypothesis regarding the boundedness of the thermoelastic coefficients. (C)2017 All rights reserved.
\end{abstract}

Keywords: Final boundary value problem, uniqueness of solution, microstretch, thermoelastic body. 2010 MSC: 74A15, 74A60, 35M30, 35Q74.

\section{Introduction}

The problem of uniqueness of solutions and other related problems have been extensively investigated in the last decade. There are a lot of studies on this topic, of which we mention a few: Brun [2], Knops and Payne [13], Levine [15], Wilkes [20], and so on. In the specific case of a problem with final boundary value, we can highlight the studies [1] by Ames and Payne, [5] by Ciarletta and Chirita and [12] by Iovane and Passarella. In general, the results are deduced by means of a joining of a Gronwall type inequality with some identities of Lagrange type. However, in the case of the paper by Koch and Lasiecka [14], the authors establish some results of backward in time uniqueness for waves in thermoelastic plates by means of appropriate Carleman estimates.

The microstretch elastic materials were first studied by Eringen in [6-8]. As we know, it is specific to this theory the fact that each material point is characterized by three deformable directors. These directors can only undergo breathing-type microdeformations in a microstretch continuum. Moreover, all the material points of this body can contract or stretch without being influenced by any rotations or translations.

\footnotetext{
*Corresponding author

Email addresses: m.marin@unitbv.ro (M. Marin), dumitru@cankaya.edu.tr (D. Baleanu), rellahi@engr.ucr.edu (R. Ellahi)
} doi:10.22436/jnsa.010.04.50 
The goal of the microstretch theory is to remove the differences which occur between experiments classical theory of elasticity. Indeed, the results of classical old elasticity prove not to be appropriate when the body's overall deformations are subject to effects of material microstructure. For instance, this happens in the case of graphite, human bones, polymers (that is, some granular bodies with large molecules), and so on.

The theory of microstretch elastic solids, as established by Eringen, was continuously studied in various papers, such as [3, 10,11]. For example, the basic results of Eringen were employed by Ciarletta in [3] for the investigation bending of plates consist of microstretch elastic material in isothermal case. Furthermore, the boundary value problem is presented with a solution of Boussinesq - Somigliana - Galerkin type by Iesan and Pompei in paper [10]. We also tackle some problems of these materials in the studies [17-19].

We want to outline that precedent papers regarding results of uniqueness of solutions or different kind of continuous dependence in elasticity or thermoelasticity are based very often on the fact that the thermoelastic coefficients or elasticity tensor are assumed be positive definite. In this regard, we recall the paper [20].

Other authors approach the same issues by using different types of energy conservation laws, means of an energy conservation law. Also, Green and Laws indicate in [9] a uniqueness result that is derived by adding certain definiteness assumptions to the restrictions arising from thermodynamics. As an exception to the rule, the uniqueness result of Brun in [2] is obtained using medium restrictions. More exactly, Brun employs a specific conservation law of energy and an identity of Lagrange type in order to establish a uniqueness result in the isothermal theory.

Our present study approaches the problem of uniqueness for solutions for the problem called backward in time, in the case of microstretch bodies. We will obtain two main results on uniqueness by means of some identities of Lagrange type in conjunction with some differential inequalities. To be more specific, we will show that under no strong hypotheses on the thermoelastic coefficients, the final boundary value problem of the microstretch thermoelasticity has sure a solution if the displacement and temperature fields satisfy no strong conditions. Namely, the conductivity tensor is assumed to be positive definite. Also, the density mass, the specific coefficient of heat and the coefficients of inertia are assumed to be strictly positive.

\section{Basic equations}

We consider that a microstretch elastic body occupies a bounded region B of the three-dimensional Euclidean space $R^{3}$. This is related to the reference configuration and a fixed system of rectangular Cartesian axes. Let $\partial B$ be the boundary of $B$ which is assumed to be a surface, piecewise smooth, surface and let $n_{i}$ be the components of the outward unit normal to the surface $\partial B$.

The material time derivative is represented by a superposed dot. The usual summation and differentiation conventions are employed and the subscripts are assumed to range over the integers $(1,2,3)$. Repeated subscripts imply summation and subscripts that have before them a comma imply partial differentiation with respect to the appropriate Cartesian coordinate. By convention, we will omit writing the spatial and time arguments functions where there is no possibility of confusion.

The motion of the body will be related to a fixed system of orthogonal Cartesian axes $O x_{i}, i=1,2,3$. Vector fields are represented by letters in boldface. The notation $v_{i}$ stands for the component $i$ of $v$, which is a vector field in the corresponding orthogonal Cartesian coordinate system.

The displacement vector field $u$ has the components $u_{i}$ and the microrotation vector field $\varphi$ has the components $\varphi_{i}$. Moreover, we consider $\omega$ to be the function of microstretch and denote by $\theta$ the temperature field which is reported to the constant temperature $T_{0}$ of the medium in its initial state.

As usual, we will use the notations $t_{i j}$ for the components of the classic stress tensor and $m_{i j}$ represents the components of the couple stress tensor over B. Also, we consider that the microstress vector has the components $\lambda_{i}$. 
For a good formalization of the problem with final boundary value for microstretch thermoelastic bodies on the interval $(-\infty, 0]$, we will systematize the basic equations. So, according to Iesan and Pompei [10], if the thermoelastic microstretch body is a homogeneous and anisotropic material, we can write the constitutive equations as follows

$$
\begin{aligned}
t_{i j} & =A_{i j r s} \varepsilon_{r s}+B_{i j r s} \mu_{r s}+D_{i j r} \gamma_{r}+a_{i j} \omega-D_{i j} \theta, \\
m_{i j} & =B_{i j s s} \varepsilon_{r s}+C_{i j s s} \mu_{r s}+E_{i j r} \gamma_{r}+b_{i j} \omega-E_{i j} \theta, \\
\lambda_{i} & =D_{r s i} \varepsilon_{r s}+E_{r s i} \mu_{r s}+C_{i j} \gamma_{j}+d_{i} \omega-F_{i} \theta, \\
\tau & =a_{i j} \varepsilon_{i j}+b_{i j} \mu_{i j}+d_{i} \gamma_{i}+m \omega-\alpha \theta, \\
\eta & =E_{i j} \varepsilon_{i j}+D_{i j} \mu_{i j}+F_{i} \gamma_{i}+\alpha \omega+\frac{a}{T_{0}} \theta, \\
q_{i} & =k_{i j} \theta_{, j} .
\end{aligned}
$$

The constitutive coefficients $A_{i j r s}, B_{i j r s}, C_{i j r s}, D_{i j r}, E_{i j r}, a_{i j}, b_{i j}, c_{i j}, d_{i}, E_{i j}, D_{i j}, F_{i}, m, \alpha, a$, and $k_{i j}$ are features for each type of material. Also, the motion equations are

$$
t_{j i, j}+f_{i}=\rho \ddot{u}_{i}, \quad m_{j i, j}+\varepsilon_{i j k} t_{j k}+g_{i}=I_{i j} \ddot{\varphi}_{j}, \quad \lambda_{i, i}-\tau+l=J \ddot{\omega},
$$

and the equation of energy is given by

$$
\mathrm{T}_{0} \dot{\eta}=\mathrm{q}_{i, i}+\mathrm{r} .
$$

Finally, the strain tensors have the components $\varepsilon_{i j}, \mu_{i j}$, and $\gamma_{i}$ and these are obtained from the geometric equations

$$
\varepsilon_{i j}=u_{j, i}+\varepsilon_{i j k} \varphi_{k}, \mu_{i j=} \varphi_{j, i}, \gamma_{i}=\omega_{, i}
$$

We must emphasize that the above equations (2.1)-(2.4) are satisfied for $(t, x) \in(-\infty, 0] \times \overline{\mathrm{B}}$. Also, in equations (2.1)-(2.4) we used some notations which have the signification that follows

- $f_{i}$ the body force;

- $g_{i}$ the body couple force;

- $\varepsilon_{i j k}$ the alternating symbol;

- $l$ the generalized external body load;

- $\tau$ the generalized internal body load;

- $\rho$ the reference constant mass density;

- $\mathrm{J}$ and $\mathrm{I}_{i j}=\mathrm{I}_{j i}$ are the coefficients of microinertia;

- $\eta$ the entropy;

- $r$ the heat supply;

- $\mathrm{q}_{i}$ heat flux vector.

Considering the geometric equations, we deduce that the constitutive elastic tensors satisfy the following relations of symmetry.

$$
A_{i j r s}=A_{r s i j}, C_{i j r s}=C_{r s i j}, C_{i j}=C_{j i}, k_{i j}=k_{j i} .
$$

Using fifth equation in (2.1), the equation of energy (2.3) get the next form

$$
-q_{i, i}+r-T_{0}\left(D_{i j} \dot{\varepsilon}_{i j}+E_{i j} \dot{\mu}_{i j}+F_{i} \dot{\gamma}_{i}+\alpha \dot{\omega}\right)=a \dot{\theta} .
$$

We denote the surface traction components by $t_{i}$, the surface couple components by $m_{i}$, the microsurface traction by $p$, and the specific heat flux by $q$ and these notions are defined by

$$
t_{i}=t_{i j} n_{j}, m_{i}=m_{i j} n_{j}, p=\lambda_{i} n_{i}, q=q_{i} n_{i} .
$$


As per usual, we denote by $n=\left(n_{i}\right)$ the unit normal of the surface $\partial B$, outward oriented.

In the following, the system of basic equations (2.1)-(2.4) will be accompanied by the final conditions

$$
\begin{aligned}
u_{i}(x, 0) & =u_{i}^{0}(x), \quad \dot{u}_{i}(x, 0)=u_{i}^{1}(x), \varphi_{i}(x, 0)=\varphi_{i}^{0}(x), \dot{\varphi}_{i}(x, 0)=\varphi_{i}^{1}(x), \\
\omega(x, 0) & =\omega_{0}(x), \quad \dot{\omega}(x, 0)=\omega_{1}(x), \theta(x, 0)=\theta_{0}(x), x \in \bar{B},
\end{aligned}
$$

and by the prescribed boundary conditions

$$
\begin{aligned}
u_{i} & =\tilde{u}_{i},(x, t) \in \partial \overline{B_{1}} \times(-\infty, 0], t_{i}=\tilde{t}_{i},(x, t) \in \partial B_{1}^{c} \times(-\infty, 0], \\
\varphi_{i} & =\tilde{\varphi}_{i},(x, t) \in \partial \overline{B_{2}} \times(-\infty, 0], m_{i}=\tilde{m}_{i},(x, t) \in \partial B_{2}^{c} \times(-\infty, 0], \\
\omega & =\tilde{\omega},(x, t) \in \partial \overline{B_{3}} \times(-\infty, 0], p=\tilde{p},(x, t) \in \partial B_{3}^{c} \times(-\infty, 0], \\
\theta & =\tilde{\theta},(x, t) \in \partial \bar{B}_{4} \times(-\infty, 0], q=\tilde{q},(x, t) \in \partial B_{4}^{c} \times(-\infty, 0] .
\end{aligned}
$$

Here $\partial \overline{B_{1}}, \partial \overline{B_{2}}, \partial \overline{B_{3}}$ and $\partial \bar{B}_{4}$ together with their complements $\partial B_{1}^{c}, \partial B_{2}^{c}, \partial B_{3}^{c}$ and $\partial B_{4}^{c}$ are parts of the boundary $\partial \mathrm{B}$ and have properties

$$
\begin{aligned}
& \partial B_{1} \cap \partial B_{1}^{c}=\partial B_{2} \cap \partial B_{2}^{c}=\partial B_{3} \cap \partial B_{3}^{c}=\partial B_{4} \cap \partial B_{4}^{c}=\emptyset, \\
& \partial \overline{B_{1}} \cup \partial B_{1}^{c}=\partial \overline{B_{2}} \cup \partial B_{2}^{c}=\partial \bar{B}_{3} \cup \partial B_{3}^{c}=\partial \bar{B}_{4} \cup \partial B_{4}^{c}=\partial B .
\end{aligned}
$$

Also, the functions $u_{i}^{0}, u_{i}^{1}, \varphi_{i}^{0}, \varphi_{i}^{1}, \omega_{0}, \omega_{1}, \theta_{0}, \tilde{u}_{i}, \tilde{t}_{i}, \tilde{\varphi}_{i}, \tilde{m}_{i}, \tilde{\omega}, \tilde{p}, \tilde{\theta}$, and $\tilde{q}$ are known in respective domains of definitions.

Regarding the assumptions of regularity, from the beginning we suppose that

(i) all the constitutive coefficients are assumed be continuously differentiable functions on the domain $\overline{\mathrm{B}}$;

(ii) the functions $\rho, I_{j k}$, and J are assumed to be continuous on the domain $\bar{B}$;

(iii) the functions $f_{i}, g_{i}, l$, and $r$ are assumed to be continuous on the cylinder $\bar{B} \times(-\infty, 0]$;

(iv) the functions $\mathfrak{u}_{0 i}, \mathfrak{u}_{1 i}, \varphi_{0 i}, \varphi_{1 i}, \omega_{0}, \omega_{1}$, and $\theta_{0}$ are assumed to be continuous on the domain $\overline{\mathrm{B}}$;

(v) the functions $\tilde{u}_{i}, \tilde{\varphi}_{i}, \tilde{\omega}$, and $\tilde{\theta}$ are assumed to be continuous on the cylinders $\partial B_{1} \times(-\infty, 0]$, $\partial \mathrm{B}_{2} \times(-\infty, 0], \partial \mathrm{B}_{3} \times(-\infty, 0]$, and $\partial \mathrm{B}_{4} \times(-\infty, 0]$, respectively;

(vi) the functions $\tilde{\mathrm{t}}_{i}, \tilde{\mathrm{m}}_{i}, \tilde{\omega}, \tilde{\mathrm{p}}$, and $\tilde{\mathrm{q}}$ are almost everywhere regular with regard to $x$ on the cylinders $\partial \mathrm{B}_{1}^{\mathrm{c}} \times(-\infty, 0], \partial \mathrm{B}_{2}^{\mathrm{c}} \times(-\infty, 0], \partial \mathrm{B}_{3}^{\mathrm{c}} \times(-\infty, 0]$, and $\partial \mathrm{B}_{4}^{\mathrm{c}} \times(-\infty, 0]$, and continuous with regard to time.

Let us denote by $(\mathcal{P F})$ the final problem consisting of equations (2.1)-(2.4), the boundary conditions (2.6) and the final conditions (2.5). The main aim of our study is to prove the uniqueness of solutions of problem $(\mathcal{P F})$ without imposing strong conditions on thermoelastic coefficients. Using a suggestion given by Ciarletta in [4], we substitute $t$ by $-t$ such that the final problem is transformed in an initial boundary value problem, denoted by (PJ). To this end, considering the constitutive equations (2.1), from (2.2) and (2.3) we are led to the system of equations that follows

$$
\begin{aligned}
\rho \ddot{u}_{i}= & \left(A_{i j r s} \varepsilon_{r s}\right)_{, j}+\left(B_{i j r s} \mu_{r s}\right)_{, j}+\left(D_{i j r} \gamma_{r}\right)_{, j}+\left(a_{i j} \omega\right)_{, j}-\left(D_{i j} \theta\right)_{, j}+f_{i}, \\
I_{i j} \ddot{\varphi}_{j}= & \left(B_{i j r s} \varepsilon_{r s}\right)_{, j}+\left(C_{i j r s} \mu_{r s}\right)_{, j}+\left(E_{i j r} \gamma_{r}\right)_{, j}+\left(b_{i j} \omega\right)_{, j}-\left(E_{i j} \theta\right)_{, j}+ \\
& +\varepsilon_{i j k}\left(A_{j k r s} \varepsilon_{r s}+B_{j k r s} \mu_{r s}+D_{j k r} \gamma_{r}+a_{j k} \omega-E_{j k} \theta\right)+g_{i}, \\
J \ddot{\omega}= & \left(D_{r s i} \varepsilon_{r s}\right)_{, i}+\left(E_{r s i} \mu_{r s}\right)_{, i}+\left(C_{i j} \gamma_{j}\right)_{, i}+\left(d_{i} \omega\right)_{, i}-\left(F_{i} \theta\right)_{, i} \\
& -a_{i j} \varepsilon_{i j}-b_{i j} \mu_{i j}-d_{i} \gamma_{i}-m \omega+\alpha \theta+l \\
-a \dot{\theta}= & \left(k_{i j} \theta_{, j}\right)_{, i}-r+T_{0}\left(E_{i j} \dot{\varepsilon}_{i j}+D_{i j} \dot{\mu}_{i j}+F_{i} \dot{\gamma}_{i}+\alpha \dot{\omega}\right),
\end{aligned}
$$

which are satisfied in cylinder $B \times(0, \infty)$. Also, the initial data receive exactly the same form as the final conditions (2.5), namely

$$
\begin{aligned}
u_{i}(x, 0) & =u_{i}^{0}(x), \quad \dot{u}_{i}(x, 0)=u_{i}^{1}(x), \varphi_{i}(x, 0)=\varphi_{i}^{0}(x), \dot{\varphi}_{i}(x, 0)=\varphi_{i}^{1}(x), \\
\omega(x, 0) & =\omega_{0}(x), \quad \dot{\omega}(x, 0)=\omega_{1}(x), \theta(x, 0)=\theta_{0}(x), x \in \bar{B},
\end{aligned}
$$


and the boundary conditions (2.6) receive the form

$$
\begin{aligned}
u_{i} & =\tilde{u}_{i},(x, t) \in \partial \overline{B_{1}} \times[0, \infty), t_{i}=\tilde{t}_{i},(x, t) \in \partial B_{1}^{c} \times[0, \infty), \\
\varphi_{i} & =\tilde{\varphi}_{i},(x, t) \in \partial \overline{B_{2}} \times[0, \infty), m_{i}=\tilde{m}_{i},(x, t) \in \partial B_{2}^{c} \times[0, \infty), \\
\omega & =\tilde{w},(x, t) \in \partial \overline{B_{3}} \times[0, \infty), p=\tilde{p},(x, t) \in \partial B_{3}^{c} \times[0, \infty), \\
\theta & =\tilde{\theta},(x, t) \in \partial \overline{B_{4}} \times[0, \infty), q=\tilde{q},(x, t) \in \partial B_{4}^{c} \times[0, \infty) .
\end{aligned}
$$

Based on these considerations, our problem with final boundary value $(\mathcal{P F})$ was transformed in the problem with initial boundary value (PJ), which consists of partial differential equations (2.7), the boundary data (2.9) and the initial data (2.8).

\section{Main result}

Because of linearity of equations (2.7) and of conditions (2.8) and (2.9) we conclude that if we consider the difference of two arbitrary solutions of the problem $(\mathcal{P J})$ then it is also a solution to this problem, but corresponding to zero loads and zero initial conditions and also zero boundary conditions. For this reason, in the following we will use the conditions (2.8) and conditions (2.9) in their homogeneous form. At first we will prove some identities which are useful in getting of main result.

Proposition 3.1. For any solution of problem (PJ) corresponding to zero loads, null initial data, and null boundary data, we have

$$
\begin{aligned}
& \int_{B}\left[\rho(x) \dot{u}_{i} \dot{u}_{i}+I_{i j} \dot{\varphi}_{i} \dot{\varphi}_{j}+J \dot{\omega}^{2}++A_{i j r s} \varepsilon_{r s} \varepsilon_{i j}+2 B_{i j r s} \varepsilon_{r s} \mu_{i j}+C_{i j r s} \mu_{r s} \mu_{i j}\right. \\
& \left.\quad+2 D_{i j r} \varepsilon_{i j} \gamma_{r}+2 E_{i j r} \mu_{i j} \gamma_{r}+C_{i j} \gamma_{i} \gamma_{j}\right] d V \\
& =-2 \int_{0}^{t} \int_{B}\left[D_{i j} \theta(s) \dot{\varepsilon}_{i j}(s)+E_{i j} \theta(s) \dot{\mu}_{i j}(s)+F_{i} \theta(s) \dot{\gamma}_{i}(s)\right] d V d s, \\
& \int_{B} \frac{1}{T_{0}} a \theta^{2}(t) d V-2 \int_{0}^{t} \int_{B} \frac{1}{T_{0}} k_{i j} \theta, i(s) \theta, j(s) d V d s \\
& =2 \int_{0}^{t} \int_{B}\left[D_{i j} \theta(s) \dot{\varepsilon}_{i j}(s)+E_{i j} \theta(s) \dot{\mu}_{i j}(s)+F_{i} \theta(s) \dot{\gamma}_{i}(s)\right] d V d s
\end{aligned}
$$

for all $\mathrm{t} \in[0, \infty)$.

Proof. Both identities are obtained by direct calculation. Elementary combinations are made between equations (2.7) and constitutive equations (2.1) and geometric equations (2.4). Then the divergence theorem is used by taking into account the null conditions.

Proposition 3.2. For any solution of problem (PJ) corresponding to zero loads, null initial data, and null boundary data, we have

$$
\begin{gathered}
\int_{B}\left[\rho(x) \dot{u}_{i} \dot{u}_{i}+I_{i j} \dot{\varphi}_{i} \dot{\varphi}_{j}+J \dot{\omega}^{2}+\frac{1}{T_{0}} a \theta^{2}+A_{i j r s} \varepsilon_{r s} \varepsilon_{i j}+2 B_{i j r s} \varepsilon_{r s} \mu_{i j}+C_{i j r s} \mu_{r s} \mu_{i j}\right. \\
\left.+2 D_{i j r} \varepsilon_{i j} \gamma_{r}+2 E_{i j r} \mu_{i j} \gamma_{r}+C_{i j} \gamma_{i} \gamma_{j}\right] d V-2 \int_{0}^{t} \int_{B} \frac{1}{T_{0}} k_{i j} \theta_{, i}(s) \theta_{, j}(s) d V d s=0, \\
\int_{B}\left[\rho(x) \dot{u}_{i} \dot{u}_{i}+I_{i j} \dot{\varphi}_{i} \dot{\varphi}_{j}+J \dot{\omega}^{2}\right] d V= \\
\int_{B}\left[A_{i j r s} \varepsilon_{r s} \varepsilon_{i j}+2 B_{i j r s} \varepsilon_{r s} \mu_{i j}+C_{i j r s} \mu_{r s} \mu_{i j}\right. \\
\left.+2 D_{i j r} \varepsilon_{i j} \gamma_{r}+2 E_{i j r} \mu_{i j} \gamma_{r}+C_{i j} \gamma_{i} \gamma_{j}+\frac{1}{T_{0}} a \theta^{2}\right] d V,
\end{gathered}
$$




$$
\int_{B}\left[\rho(x) \dot{u}_{i} \dot{u}_{i}+I_{i j} \dot{\varphi}_{i} \dot{\varphi}_{j}+J \dot{\omega}^{2}\right] d V=\int_{0}^{t} \int_{B} \frac{1}{T_{0}} k_{i j} \theta_{, i}(s) \theta, j(s) d V d s
$$

for all $\mathrm{t} \in[0, \infty)$.

Proof. Identity (3.3) can be achieved by adding term by term the identities (3.1) and (3.2). Identity (3.4) is obtained by direct calculation, with combinations between equations (2.7) and constitutive equations (2.1) and geometric equations (2.4). Then the divergence theorem is used by taking into account the null conditions. Finally, identity (3.5) is obtained with the help of (3.3) and (3.4).

To establish that the solution of problem $(\mathcal{P J})$ is unique, we suppose that $a$ is strictly positive in B (a being the specific heat). Also, we need to assume that

$$
I_{i j} \xi_{i} \xi_{j} \geqslant m_{0} \xi_{i} \xi_{i}, \forall \xi_{i}
$$

where $\mathrm{m}_{0}$ is a positive constant.

According to the second law of thermodynamics, we have that the tensor of thermal conductivity $k_{i j}$ is a positive definite one, that is, there exists a constant $k_{0}>0$ so that

$$
k_{i j} \xi_{i} \xi_{j} \geqslant k_{0} \xi_{i} \xi_{i}, \forall \xi_{i} .
$$

Now we can state the main result of uniqueness.

Theorem 3.3. We suppose the following assumptions are met

i) $\rho>0, \mathrm{~J}>0, \mathrm{I}_{\mathrm{ij}}$ satisfies (3.6);

ii) the tensor $k_{i j}$ is positive definite and the elastic coefficients are positive semi-definite tensors;

iii) the specific coefficient of heat, $\mathrm{a}$, is assumed to be strictly positive;

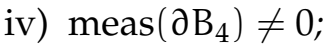

v) there are constants $M_{1}$ and $c_{0}>0$ so that

$$
\int_{0}^{t} \int_{B} \frac{1}{T_{0}} k_{i j} \theta_{, i}(s) \theta_{, j}(s) d V d s \leqslant M_{1}^{2} e^{c_{0} t}, \forall t \in[0, \infty)
$$

Then the problem (PJ) has at most one solution.

Proof. If $\left(u_{i}(t, x), \varphi_{i}(t, x), w(t, x), \theta(t, x)\right)$ is a solution of problem $(\mathcal{P J})$, that is, that corresponds to null loads and null initial data and also null boundary data, then it is necessary to prove that

$$
\left(u_{i}, \varphi_{i}, \omega, \theta\right)(t, x)=0, \forall(t, x) \in[0, \infty) \times B .
$$

To this aim we will use the identities (3.1)-(3.5). As a first step, from (3.4) and (3.5) we lead to

$$
\begin{gathered}
\int_{B}\left[A_{i j r s} \varepsilon_{r s}(t) \varepsilon_{i j}(t)+2 B_{i j r s} \varepsilon_{r s}(t) \mu_{i j}(t)+C_{i j r s} \mu_{r s}(t) \mu_{i j}(t)+2 D_{i j r} \varepsilon_{i j}(t) \gamma_{r}(t)+2 E_{i j r} \mu_{i j}(t) \gamma_{r}(t)\right. \\
\left.\quad+C_{i j} \gamma_{i}(t) \gamma_{j}(t)+\frac{1}{T_{0}} a \theta^{2}(t)\right] d V=\int_{0}^{t} \int_{B} \frac{1}{T_{0}} k_{i j} \theta_{, i}(s) \theta, j(s) d V d s,
\end{gathered}
$$

and this identity holds true for all $t \in[0, \infty)$.

Assuming that all elasticity tensors are negative semi-definite, from (3.9) we obtain

$$
\int_{0}^{t} \int_{B} \frac{1}{T_{0}} k_{i j} \theta, i(s) \theta, j(s) d V d s \leqslant \int_{B} \frac{1}{T_{0}} a \theta^{2}(t) d V, \forall t \in[0, \infty) .
$$


On the other hand, if we denote by $\lambda_{0}>0$ the minimum eigenvalue of the conductivity tensor, then taking into account that meas $\left(\partial \mathrm{B}_{4}\right) \neq 0$, we obtain

$$
\int_{B} \theta, i(s) \theta, j(s) d V \geqslant \lambda_{0} \int_{B} \theta^{2}(t) d V
$$

Let us introduce the notation

$$
w^{2}=\frac{\sup _{\overline{\mathrm{B}}} \mathrm{a}}{\lambda_{0} \mathrm{k}_{0}},
$$

in which $k_{0}$ has been introduced in (3.7).

Now combine inequalities (3.7), (3.10), and (3.11) so we are led to

$$
\int_{0}^{t} \int_{B} \frac{1}{T_{0}} k_{i j} \theta, i(s) \theta, j(s) d V d s \leqslant w^{2} \int_{B} \frac{1}{T_{0}} k_{i j} \theta, i(s) \theta, j(s) d V, \forall t \in[0, \infty) .
$$

Obviously, this inequality is of the form

$$
f^{2}(t) \leqslant 2 w^{2} f(t) \dot{f}(t), \forall t \in[0, \infty),
$$

in which, clearly, function $f(t)$ is

$$
f^{2}(t)=\int_{0}^{t} \int_{B} \frac{1}{T_{0}} k_{i j} \theta, i(s) \theta, j(s) d V d s, \forall t \in[0, \infty) .
$$

Let us prove that $f(t) \leqslant 0, \forall t \in[0, \infty)$. Suppose by contradiction that there exists $s>0$ such that $f(s)>0$ then we have

$$
f(t)>0, \forall t \in[s, \infty) .
$$

Taking into account the property of $f$, from (3.12), we deduce

$$
\frac{\mathrm{d}}{\mathrm{dt}}\left[\mathrm{f}(\mathrm{t}) \mathrm{e}^{-\mathrm{t} /\left(2 w^{2}\right)}\right] \geqslant 0, \forall \mathrm{t} \in[s, \infty),
$$

from where, through integration, we are led to

$$
f(s) e^{-s /\left(2 w^{2}\right)} \leqslant f(t) e^{-t /\left(2 w^{2}\right)} \leqslant \lim _{t \rightarrow \infty}\left[f(t) e^{-t /\left(2 w^{2}\right)}\right], \forall t \in[s, \infty) .
$$

Now, by choosing of $c_{0}$ in inequality (3.8) so that

$$
0 \leqslant \mathrm{c}_{0}<\frac{1}{w^{2}}
$$

we will find that

$$
\lim _{t \rightarrow \infty}\left[f(t) e^{-t /\left(2 w^{2}\right)}\right]=0 .
$$

This relation together with (3.15) leads to the conclusion

$$
f(t)=0, \forall t \in[s, \infty),
$$

that contradicts the inequality (3.14).

Thus we concluded that $f(t)=0, \forall t \in[0, \infty)$, such that from (3.13) we obtain

$$
\theta, i(t, x)=0, \text { in }[0, \infty) \times B
$$


and, because, we get

$$
\theta(t, x)=0 \text {, in }[0, \infty) \times \overline{\mathrm{B}} .
$$

Finally, from (3.16) and (3.5), taking into account the null initial conditions, we deduce

$$
u_{i}=0, \varphi_{i}=0, w=0 \text {, in }[0, \infty) \times \bar{B} .
$$

So, the proof of Theorem 3.3 is complete.

The following theorem is also dedicated to the uniqueness of the solution, but we abandon the hypotheses that the elasticity tensors are negative semi-definite and meas $\left(\partial \mathrm{B}_{4}\right) \neq 0$.

Theorem 3.4. We assume that the following assumptions are met

i) $a>0, \rho>0, J>0, I_{i j}$ satisfies (3.6);

ii) $\mathrm{k}_{i j}$ is a positive definite tensor and meas $\left(\partial \mathrm{B}_{4}\right)=0$;

iii) the conductivity tensor $k_{i j}$ satisfies the additional hypothesis

$$
\lim _{t \rightarrow \infty} \int_{0}^{t} \int_{0}^{s} \int_{B} \frac{1}{T_{0}} k_{i j} \theta, i(\tau) \theta, j(\tau) d V d \tau d s=0
$$

Then the problem (PJ) has at most one solution.

Proof. In the context of the coupled theory of thermoelasticity, we have

$$
m=\left(D_{i j} D_{i j}+E_{i j} E_{i j}+F_{i} F_{i}\right)^{1 / 2}>0 .
$$

Also, we set

$$
m^{*}=\left(D_{i j, k} D_{i j, k}+E_{i j, k} E_{i j, k}+F_{i, k} F_{i, k}\right)^{1 / 2} \geqslant 0 .
$$

With the help of the smallest eigenvalue defined in (3.11), we introduce the constant $M_{2}$ by

$$
M_{2}=\max \left\{\sup _{\bar{B}} \frac{m}{\sqrt{\rho}}, \sup _{\bar{B}} \frac{m^{*}}{\sqrt{\lambda_{0} \rho}}\right\} .
$$

Then, we apply the arithmetic-geometric mean inequality and the Cauchy-Schwarz inequality in the right-hand side of the identity (3.1), so we find that for all $t \in[0, \infty)$ the following estimate holds true

$$
\begin{aligned}
& -2 \int_{0}^{t} \int_{B}\left[D_{i j} \theta(s) \dot{\varepsilon}_{i j}(s)+E_{i j} \theta(s) \dot{\mu}_{i j}(s)+F_{i} \theta(s) \dot{\gamma}_{i}(s)\right] d V d s \\
& \leqslant \frac{M_{2}}{\varepsilon \sqrt{k_{0}}} \int_{B}\left[\rho(x) \dot{u}_{i}(t) \dot{u}_{i}(t)+I_{i j} \dot{\varphi}_{i}(t) \dot{\varphi}_{j}(t)+J \dot{\omega}^{2}(t)\right] d V \\
& \quad+\frac{4 T_{0} M_{2}}{\sqrt{k_{0}}} \varepsilon \int_{0}^{t} \int_{B} \frac{1}{T_{0}} k_{i j} \theta, i(s) \theta, j(s) d V d s,
\end{aligned}
$$

where we donoted by $\varepsilon$ a positive constant which is convenient chosen for the inequality of arithmeticgeometric mean.

Now, we shall use (3.2) taking into account (3.5) and (3.18) so that we get

$$
\begin{aligned}
\left(2-\frac{4 T_{0} M_{2}}{\sqrt{k_{0}}} \varepsilon\right) & \int_{B}\left[\rho \dot{u}_{i} \dot{u}_{i}+I_{i j} \dot{\varphi}_{i} \dot{\varphi}_{j}+J \dot{\omega}^{2}\right] d V \\
& \leqslant \int_{B} \frac{1}{T_{0}} a \theta^{2} d V+\frac{M_{2}}{\varepsilon \sqrt{k_{0}}} \int_{0}^{t} \int_{B}\left[\rho \dot{u}_{i}(s) \dot{u}_{i}(s)+I_{i j} \dot{\varphi}_{i}(s) \dot{\varphi}_{j}(s)+J \dot{\omega}^{2}(s)\right] d V d s,
\end{aligned}
$$


If we use the notations

$$
\alpha(t)=\int_{B}\left[\rho \dot{u}_{i} \dot{u}_{i}+I_{i j} \dot{\varphi}_{i} \dot{\varphi}_{j}+J \dot{\omega}^{2}\right] d V, \quad \beta(t)=\int_{B} \frac{1}{T_{0}} a \theta^{2} d V, M_{0}=\frac{4 T_{0} M_{2}^{2}}{k_{0}},
$$

and choose for $\varepsilon$ the value

$$
\varepsilon=\frac{\sqrt{\mathrm{k}_{0}}}{4 \mathrm{~T}_{0} \mathrm{M}_{2}}
$$

then the inequality (3.19) can be rewritten formally in a very simple form, namely

$$
\alpha(t) \leqslant \beta(t)+M_{0} \int_{0}^{t} \alpha(s) d s .
$$

Also, with the help of notation

$$
\gamma(\mathrm{t})=e^{-\mathrm{M}_{0} \mathrm{t}} \mathrm{M}_{0} \int_{0}^{\mathrm{t}} \alpha(\mathrm{s}) \mathrm{ds},
$$

using (3.21), we immediately deduce that

$$
\dot{\gamma}(t)=M_{0} e^{-M_{0} t}\left[\alpha(t)-M_{0} \int_{0}^{t} \alpha(s) d s\right] \leqslant M_{0} e^{-M_{0} t} \beta(t) .
$$

As such, from (3.22) and (3.23) it results

$$
\gamma(t) \leqslant M_{0} \int_{0}^{t} e^{-M_{0} s} \beta(s) d s
$$

for all $t \in[0, \infty)$.

As such, from (3.22) and (3.23) for all $t \in[0, \infty)$ we obtain the estimate

$$
\int_{0}^{t} \alpha(s) d s \leqslant \int_{0}^{t} e^{-M_{0}(s-t)} \beta(s) d s .
$$

If we take into account (3.7) and (3.11), from (3.20) we deduce

$$
\beta(t) \leqslant \frac{\sup _{\bar{B}} a}{\lambda k_{0}} \int_{B} \frac{1}{T_{0}} k_{i j} \theta, i(\tau) \theta_{, j}(\tau) d V,
$$

and this estimate introduced in (3.24) leads to

$$
\int_{0}^{t} \alpha(s) d s \leqslant \frac{\sup _{\bar{B}} a}{\lambda k_{0}} e^{M_{0} t} \alpha(t), \forall t \in[0, \infty) .
$$

On the other hand, if we introduce the notation

$$
\psi(t)=\left(\int_{0}^{t} \alpha(s) d s\right)^{1 / 2}, t \in[0, \infty),
$$

then the inequality (3.25) can be rewritten in the form

$$
\psi^{2}(\mathrm{t}) \leqslant \frac{2 \sup _{\overline{\mathrm{B}}} \mathrm{a}}{\lambda \mathrm{k}_{0}} e^{\mathrm{M}_{0} \mathrm{t}} \psi(\mathrm{t}) \dot{\psi}(\mathrm{t}), \forall \mathrm{t} \in[0, \infty) .
$$

In the following we will prove that

$$
\psi(\mathrm{t})=0, \forall \mathrm{t} \in[0, \infty) .
$$


Suppose by contradiction that we find a $\tau \in(0, \infty)$ so that $\psi(\tau)>0$. Then we deduce

$$
\psi(t)>0, \forall t \in[\tau, \infty) .
$$

If we set

$$
\xi(t)=\frac{\lambda k_{0}}{2 M_{0} \sup _{\bar{B}} a}\left(e^{-M_{0} t}-1\right),
$$

then from (3.26) we obtain

$$
\frac{d}{d t}\left[e^{\xi(t)} \psi(t)\right] \geqslant 0, \forall t \in[\tau, \infty),
$$

so that for all $t \in[\tau, \infty)$ we can write

$$
e^{\xi(t)} \psi(\tau) \leqslant e^{\xi(t)} \psi(t) \leqslant \lim _{t \rightarrow \infty}\left[e^{\xi(t)} \psi(t)\right] .
$$

On the other hand, taking into account the hypothesis (3.17) and notation (3.20) we are led to

$$
\lim _{t \rightarrow \infty}\left[e^{\xi(t)} \psi(t)\right]=e^{-\frac{\lambda k_{0}}{2 M_{0} \sup _{\bar{B}} a}}\left[\lim _{t \rightarrow \infty} \int_{0}^{t} \int_{0}^{s} \int_{B} \frac{1}{T_{0}} k_{i j} \theta, i(\tau) \theta, j(\tau) d V d \tau d s\right]^{1 / 2}=0 .
$$

Clearly, from (3.29) it results that

$$
\psi(t)=0, \forall t \in[\tau, \infty),
$$

which is in contradiction with (3.28).

Therefore we infer that only the situation (3.27) is possible. In accordance with (3.27), from (3.20) we obtain

$$
\dot{\mathrm{u}}_{\mathrm{i}}(\mathrm{t}, x)=0, \dot{\varphi}_{i}(\mathrm{t}, x)=0, \dot{\mathrm{w}}(\mathrm{t}, \mathrm{x})=0, \text { in }[0, \infty) \times \overline{\mathrm{B}},
$$

so that remembering that we consider zero initial data, we are led to

$$
u_{i}=0, \varphi_{i}=0, w=0, \forall(t, x) \in[0, \infty) \times \overline{\mathrm{B}} .
$$

Finally, the hypothesis $a>0$ in $\bar{B}$ and inequality (3.11) assure that

$$
\theta=0, \forall(t, x) \in[0, \infty) \times \bar{B},
$$

and the proof of Theorem 3.4 is complete.

\section{Conclusion}

We want to emphasize that the theorems of uniqueness were proved even if we give up any conservation law of energy. Also, we do not recourse to any boundedness assumptions on the coefficients of elasticity. Our results are obtained under the assumptions that the density mass, the coefficients of microinertia and the specific heat are strictly positive. We also use the hypothesis that the conductivity tensor is positive definite. In various other papers, these results are obtained by assuming some stronger restrictions.

\section{Acknowledgment}

The authors would like to thank the reviewers for careful reading the paper and giving constructive comments, which improved the manuscript substantially. 


\section{References}

[1] K. A. Ames, L. E. Payne, Stabilizing solutions of the equations of dynamical linear thermoelasticity backward in time, Stability Appl. Anal. Contin. Media, 1 (1991), 243-260. 1

[2] L. Brun, Méthodes énergétiques dans les systémes évolutifs linéaires, II, Théorémes d'unicité, (French) J. Mécanique, 8 (1969), 167-192. 1

[3] M. Ciarletta, On the bending of microstretch elastic plates, Internat. J. Engrg. Sci., 37 (1999), 1309-1318. 1

[4] M. Ciarletta, On the uniqueness and continuous dependence of solutions in dynamical thermoelasticity backward in time, J. Thermal Stresses, 25 (2002), 969-984. 2

[5] M. Ciarletta, S. Chirita, Spatial behavior in linear thermoelasticity backward in time, Proceedings of the Fourth International Congress on Thermal Stresses, Osaka, Japan, Osaka Prefecture University, (2001), 485-488. 1

[6] A. C. Eringen, Theory of micromorphic materials with memory, Internat. J. Engrg. Sci., 10 (1972), 623-641. 1

[7] A. C. Eringen, Theory of thermo-microstretch elastic solids, Internat. J. Engrg. Sci., 28 (1990), 1291-1301.

[8] A. C. Eringen, Microcontinuum field theories, I, Foundations and solids, Springer-Verlag, New York, (1999). 1

[9] A. E. Green, N. Laws, On the entropy production inequality, Arch. Rational Mech. Anal., 45 (1972), 47-53. 1

[10] D. Ieşan, A. Pompei, On the equilibrium theory of microstretch elastic solids, Internat. J. Engrg. Sci., 33 (1995), $399-410$. 1,2

[11] D. Ieşan, R. Quintanilla, Thermal stresses in microstretch elastic plates, Internat. J. Engrg. Sci., 43 (2005), 885-907. 1

[12] G. Iovane, F. Passarella, Spatial behavior in dynamical thermoelasticity backward in time for porous media, J. Thermal Stresses, 27 (2004), 97-109. 1

[13] R. J. Knops, L. E. Payne, On uniqueness and continuous dependence in dynamical problems of linear thermoelasticity, Int. J. Solids Struct., 6 (1970), 1173-1184. 1

[14] H. Koch, I. Lasiecka, Backward uniqueness in linear thermoelasticity with time and space variable coefficients, Functional analysis and evolution equations, Birkhüser, Basel, (2008), 389-403. 1

[15] H. A. Levine, On a theorem of Knops and Payne in dynamical linear thermo-elasticity, Arch. Rational Mech. Anal., 38 (1970), 290-307. 1

[16] M. Marin, Some basic theorems in elastostatics of micropolar materials with voids, J. Comput. Appl. Math., 70 (1996), 115-126.

[17] M. Marin, A domain of influence theorem for microstretch elastic materials, Nonlinear Anal. Real World Appl., 11 (2010), 3446-3452. 1

[18] M. Marin, M. Lupu, On harmonic vibrations in thermoelasticity of micropolar bodies, J. Vib. Control, 4 (1998), 507-518.

[19] K. Sharma, M. Marin, Reflection and transmission of waves from imperfect boundary between two heat conducting micropolar thermoelastic solids, An. St. Univ. Ovidius Constanta, 22 (2014), 151-175. 1

[20] N. S. Wilkes, Continuous dependence and instability in linear thermoelasticity, SIAM J. Math. Anal., 11 (1980), $292-299$. 1 